\title{
Skeletal Troponin I Measurement
}

National Cancer Institute

\section{Source}

National Cancer Institute. Skeletal Troponin I Measurement. NCI Thesaurus. Code C135443.

The determination of the amount of total skeletal troponin I in a biological sample. 